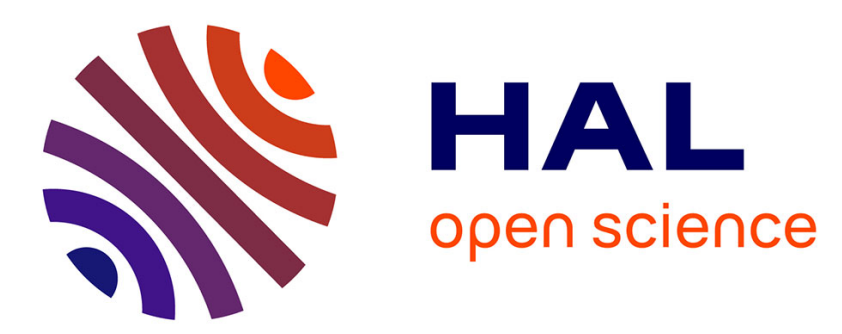

\title{
Réseaux migratoires et structures de pouvoir : la Church of Pentecost du Ghana face aux communautés francophones
}

\author{
Sandra Fancello
}

\section{> To cite this version:}

Sandra Fancello. Réseaux migratoires et structures de pouvoir : la Church of Pentecost du Ghana face aux communautés francophones. Institut Français de Recherche en Afrique. Entreprises religieuses transnationales en Afrique de l'Ouest, IFRA-Karthala, pp.111-134, 2005. halshs-00004665

\section{HAL Id: halshs-00004665 \\ https://shs.hal.science/halshs-00004665}

Submitted on 10 Jan 2008

HAL is a multi-disciplinary open access archive for the deposit and dissemination of scientific research documents, whether they are published or not. The documents may come from teaching and research institutions in France or abroad, or from public or private research centers.
L'archive ouverte pluridisciplinaire HAL, est destinée au dépôt et à la diffusion de documents scientifiques de niveau recherche, publiés ou non, émanant des établissements d'enseignement et de recherche français ou étrangers, des laboratoires publics ou privés. 


\section{Réseaux migratoires et structures de pouvoir : la Church of Pentecost du Ghana face aux communautés francophones ${ }^{*}$}

Sandra FANCELLO

L'Église de Pentecôte, originaire du Ghana et aujourd'hui implantée dans près de cinquante pays dont vingt cinq pays africains, est travaillée par une série de débats et de controverses concernant la distribution de l'autorité, notamment dans ses rapports aux autres communautés africaines, et la place faite aux cultures locales. Tandis que des pays comme la Côte d'Ivoire ou le Togo ont progressivement adopté la langue française comme langue liturgique, au Ghana, le débat que soulève la formation d'assemblées en français et en anglais porte sur le statut de ces assemblées au sein d'une Église qui se pense à la fois «indigène » et transnationale. De même, cette Église apparaît comme l'une des plus étroitement liées au débat concernant le statut des camps de prière, de guérison et de délivrance, constitués en véritables institutions au sein même de l'Église qui peine à les soumettre à son autorité. Les conflits de leadership qui surgissent en marge de la structure hiérarchique sont à l'origine de plusieurs dissidences, au Ghana comme en Côte-d'Ivoire et au Togo. A partir d'une série d'enquêtes qui nous ont permis d'évoluer au sein d'un réseau d'Église, nous nous sommes attachée à la compréhension de ces débats et controverses qui ébranlent l'Église de Pentecôte dans ses modes de gestion des conflits, la distribution de l'autorité, ses stratégies d'implantation à l'étranger (Burkina Faso, Côte-d'Ivoire, Togo) ainsi que dans ses rapports aux autres communautés africaines, notamment francophones.

\footnotetext{
* in Fourchard L. Mary A. Otayek R. (éds.), Entreprises religieuses et réseaux transnationaux en Afrique de l'Ouest, Paris, IFRA-KARTHALA, 2005, pp. 113-136
} 


\section{Les relais de l'expansion missionnaire}

La Church of Pentecost du Ghana fait partie des premières Églises issues du mouvement pentecôtiste ghanéen. Elle fut fondée par un missionnaire écossais, James McKeown, envoyé au Ghana en 1937 par l'Église Apostolique britannique (Bradford). A la suite d'une dissidence avec cette Église en 1953, le missionnaire crée sa propre Église, qui deviendra la Church of Pentecost à partir de 1962. Cette Église serait aujourd'hui la plus importante dénomination pentecôtiste du Ghana (Gifford, 1998 : 96 ; ter Haar, 1994 : 225). L'expansion de cette Église en Afrique de l'Ouest repose sur la migration des pêcheurs ghanéens (fishermen) parfois convertis, et qui deviennent des «migrantsmissionnaires ». Ces derniers forment bientôt les premiers groupes de prière qui seront la base de l'implantation de l'Église de Pentecôte dans ces pays de migration, principalement la Côte-d'Ivoire, le Togo et le Bénin avec quelques extensions mineures dans les pays anglophones comme le Nigeria, le Liberia et la Sierra Leone. Ainsi, dans un premier temps, l'usage de la langue twi comme langue liturgique fut maintenu, y compris hors du Ghana. L'Église reste ainsi très fortement marquée par son identité ethno-nationale. Malgré son expansion rapide en direction des pays côtiers, la progression de l'Église est ralentie dans le nord du Ghana, à la frontière avec le Burkina Faso. Les points d'implantation les plus importants restent concentrés dans la zone de peuplement akan. La proximité historique et géographique des Ashanti du Ghana, avec les Agni de Côte-d'Ivoire d'une part et les Éwé du Ghana et du Togo d'autre part, a permis une première expansion rapide de cette Église sur un axe côtier qui va d'Abidjan à Lomé, aujourd'hui étendu à Cotonou et Lagos.

En 1970, la Church of Pentecost comptait quatre principales extensions à l'extérieur du Ghana: le Liberia, le Togo-Bénin alors regroupés et de loin la mission la plus importante, la Haute-Volta (actuel Burkina Faso) et la Côte-d'Ivoire. Les régions du «nord », à commencer par le nord du Ghana, mais également des pays comme le Burkina Faso, le Mali et le Niger, sont plus difficiles à atteindre pour des pasteurs essentiellement Ashanti et peu enclins à la délégation de pouvoirs à des pasteurs issus d'autres groupes ethniques. Ainsi, dans un premier temps, l'expansion missionnaire de cette Église, portée par les migrants, se fit essentiellement dans l'espace de la migration ghanéenne et ne se heurta pas immédiatement aux résistances autochtones, compte tenu de l'importance des communautés ghanéennes dans les pays côtiers du Golfe de Guinée. De cette expansion résulta la formation d'Églises nationales ou «missions extérieures » (vues du Ghana), reliées entre elles par l'Église centrale où se retrouvent régulièrement tous les pasteurs et les 
missionnaires de l'Église. Le siège de l'Église, installé à Accra, est à la fois un foyer de tensions, de production et de diffusion des objets de débats. Les relations avec les «missions extérieures» reposent sur une logique d'aller-retour entre le foyer ghanéen, les conflits émergeants dans les missions et les éléments de réponses ou directives apportées par l'Église centrale, toujours en étroite relation avec ses agents locaux. Parmi les étapes de l'expansion missionnaire de cette Église, il convient donc de distinguer les terres «de migration » comme la Côte-d'Ivoire des terres «d'implantation» comme le Burkina Faso où, selon un mouvement inverse, l'Église fut importée très tôt par des pasteurs burkinabé venus au Ghana dès la fin des années 1950 où ils s'affilièrent au groupe de James McKeown. La Côte-d'Ivoire, où l'Église de Pentecôte fut officiellement enregistrée en 1966 par les missionnaires ghanéens, est aujourd'hui le deuxième pays après le Ghana où l'implantation de l'Église est la plus importante numériquement et le seul pays avec le Ghana à envoyer des missionnaires à l'étranger, notamment vers les pays francophones d'Afrique et d'Europe.

Paradoxalement, les pays anglophones ouest-africains tels que le Nigeria, le Liberia et la Sierra Leone ne représentent pas des zones d'implantation importantes de la Church of Pentecost. Pas plus que l'Afrique de l'est (Kenya, Tanzanie) où l'Église compte peu d'assemblées. Encore moins en Afrique centrale (Zimbabwe, Zambie et le Malawi où l'entrée de l'Église date de 1994) et en Afrique du sud (Afrique du Sud, Botswana qui sont aussi des implantations récentes, respectivement 1991 et 1993) où l'Église totalise moins de cinq cent fidèles. L'expansion de la Church of Pentecost semble si étroitement liée à la migration ghanéenne que la zone d'implantation géographique de l'Église reste concentrée dans les pays limitrophes du Ghana en dépit du fait qu'il s'agit essentiellement de pays francophones. Le Nigeria, qui fut l'une des destinations de migration ghanéenne, expulsa une grande partie de la population migrante au début des années 1980 dans le cadre de l'Aliens Compliance Order (1982) et réduisit à néant l'effectif de fidèles de la Church of Pentecost qui y était enregistrée depuis 1978. Selon C. Leonard (1989), l’Église faillit disparaître complètement dans ce pays si les mouvements de population n'avaient pas progressivement repris. Ces périodes de troubles politiques ont eu pour effet de ralentir la croissance de l'Église dans certains pays, ce qui explique que le Nigeria compte aujourd'hui moins de quatre mille fidèles (Rapport missionnaire, 2002). Au Liberia, autre pays d'immigration des commerçants ghanéens, où la Church of Pentecost, présente depuis le début des années soixante-dix, aurait connu une croissance «phénoménale» selon C. Leonard (1989: 151), la communauté de fidèles s'élevait à 1300 membres en 1986. La guerre civile qui éclata au début des années 1990 dispersa la communauté de fidèles et 
les missionnaires ghanéens ${ }^{1}$. En 2002, l'Église ghanéenne comptait près de six mille fidèles. De la présence de l'Église au Liberia résulta son extension en Sierra Leone au milieu des années 1980.

La première contradiction à laquelle l'Église dut faire face concerne ses stratégies d'implantation dans les pays voisins du Ghana. Orientant son expansion d'abord en suivant les routes migratoires des communautés ghanéennes à l'étranger (Côte-d'Ivoire, Togo), l'Église adopte progressivement une stratégie d'évangélisation des populations autochtones en recrutant des agents locaux, lesquels ouvrent bientôt des assemblées dans les villes et villages où ils ont d'emblée recours aux langues vernaculaires comme langue liturgique. Progressivement, alors qu'émerge dans chacun de ces pays une élite locale d'agents autochtones, se développent des revendications nationalistes et autonomistes, sous des formes différentes d'un pays à l'autre et parfois à plusieurs années d'intervalle. Tandis que l'élite des dirigeants ghanéens cherche à maintenir une identification forte à une Église qui fait peu de concessions aux populations locales, les contradictions de cette politique identitaire se révèlent dans les affrontements entre quelques leaders issus de pays francophones et la direction ghanéenne. A leurs revendications, l'Église centrale oppose le plus souvent une décision autoritaire par le biais du Conseil Exécutif International. Mais les tensions parfois très fortes et les crises réelles qu'a suscité la politique de la Church of Pentecost du Ghana dans les missions extérieures ont forcé le siège à céder devant le fait accompli et l'ont progressivement amené à assouplir les modalités de contrôle et de soumission des Églises nationales. Ces conflits reposent en partie sur une ambition d'expansion transnationale paradoxalement mêlée à une volonté de contrôle du pouvoir sans partage. Notre interrogation porte donc sur trois points essentiels : $1^{\circ}$ ) compte tenu de la force de l'affirmation identitaire de cette Église, paradoxalement mêlée à une volonté d'expansion transnationale, quels relais se donnent les pasteurs Ashanti auprès des autres communautés africaines dans la confrontation avec la francophonie ouest-africaine, à l'extérieur de ses frontières (pays limitrophes) aussi bien qu'à l'intérieur du Ghana (concernant les migrants francophones). $2^{\circ}$ ) Dans quelle mesure et en quels termes les tensions et débats qui surgissent au sein de l'Église, se répercutent à l'intérieur du réseau, du siège aux «missions extérieures » ou inversement. $3^{\circ}$ ) Compte tenu de la centralisation de l'autorité, quels sont ou peuvent être les modes de gestion des conflits locaux. De ce point de vue, les assemblées en

\footnotetext{
1 Dans son livre, Rescapé de Flamingo, le camp du non-retour (1994), l'actuel Président de la Church of Pentecost International, l'Apôtre M. K. Ntumy, fait le récit de sa détention dans un camp de réfugiés à la fin de l'année 1990 alors qu'il était missionnaire dans le pays depuis 1988 .
} 
français et en anglais, ainsi que les camps de guérison, en tant qu'espaces minoritaires en marge de l'Église, se présentent comme des analyseurs des tensions internes, et constituent à leur tour, un réseau parallèle, marginal et informel entre leurs agents. Les actuels leaders de ces assemblées, tout comme ceux des camps, disposent d'un charisme qui ne cadre pas avec les modes de reconnaissance de l'Église, en tant qu'institution. Le statut de ces «agents charismatiques » est à l'origine de plusieurs conflits et dissidences qui divisent l'Église aujourd'hui. Quels réseaux transnationaux se tissent alors à partir de ces deux espaces communautaires et quelles sont les stratégies de résolution des conflits mises en œuvre par l'Église face à ces sphères de pouvoir qui lui échappent?

Concernant les relais de cette Église dans les pays voisins, les pasteurs Éwé furent les premiers et les plus proches auxiliaires des missionnaires ghanéens de la Church of Pentecost, comme le sont aujourd'hui les pasteurs ivoiriens (du groupe Agni). Mais l'association avec les pasteurs ivoiriens apparaît comme une étape récente dans les relations de l'élite Ashanti avec les pasteurs «autochtones» des missions extérieures. Jusqu'au début des années 1990, le Togo était en effet la mission la plus importante après le Ghana, et le premier relais francophone des pasteurs ghanéens. Une importante dissidence, qui mettait déjà en cause la gestion autoritaire du siège ghanéen, a divisé l'Église de Pentecôte du Togo et considérablement réduit son effectif, entamant ainsi ses capacités d'expansion autant que ses ressources ${ }^{2}$. Cette collaboration étroite s'était construite, comme pour les Agni de Côte-d' Ivoire aujourd'hui, sur la base d'une proximité géographique, culturelle et linguistique des deux groupes. De la collaboration avec les pasteurs Éwé résulta l'implantation de l'Église de Pentecôte à l'est du Ghana ainsi qu'au sud du Togo au début des années cinquante, quasi simultanément avec la Côte d'Ivoire, le Bénin et le Burkina Faso. Les pasteurs togolais ont ainsi largement contribué à l'implantation de cette Église dans les pays francophones limitrophes du

2 Les entretiens réalisés auprès des Apôtres B.Y. Apedo et P.K. Mensah (respectivement Présidents de l'Église de Pentecôte du Togo de 1978 à 1986 et de 1986 à 1990), ainsi que des pasteur Balo Kwasivi et Abalo Kemeli, (appartenant tous deux aujourd'hui à l'Église de Pentecôte dissidente), permettent de penser à ce propos que la lutte pour la reconnaissance de la souveraineté des pasteurs togolais dans la gestion de l'Église nationale, eu égard à leur engagement missionnaire auprès des pasteurs ghanéens, fut l'une des causes de la dissidence de 1991 qui se traduisit par la prise d'autonomie de l'Église de Pentecôte du Togo. La majorité des fidèles et pasteurs togolais aurait rejoint l'Église «nationale » tandis que ceux restés fidèles à la Church of Pentecost du Ghana ont de plus été dépossédés des temples et terrains précédemment acquis par les pasteurs togolais. Nous savons par ailleurs que le conflit qui opposa les deux Églises ayant conservé la même dénomination fit l'objet d'une décision de justice qui a contraint l'Église de Pentecôte (ghanéenne) à changer de nom. Elle aurait adopté depuis peu le nom d' «Église de Pentecôte Internationale ». 
Ghana. Il furent, dans ce contexte, les premiers interprètes des dirigeants ghanéens (peu d'entre eux, encore aujourd'hui, maîtrisent la langue française). Ces expansions collatérales s'inscrivent par ailleurs dans une stratégie missionnaire désignée «Operation Next Door», selon laquelle depuis chaque pays où l'Église est implantée, celle-ci cherche à s'étendre dans le pays voisin, à y implanter une Église à son tour, comme une extension d'elle-même, et à l'assister jusqu'à ce que la jeune Église soit elle-même en mesure de parvenir à son autonomie et d'accueillir un missionnaire résident sur ses fonds propres (Ntumy, 2000: 30). Cette stratégie fut mise en œuvre par exemple, dans le cas du Niger dont la première mission fut à l'initiative et à la charge de l'Église de Pentecôte du Burkina Faso, avant que la nouvelle mission au Niger puisse accueillir un missionnaire.

\section{La mission « inversée »}

La politique missionnaire officielle de l'Église de Pentecôte ne consiste pas, contrairement à ce que la réalité donne à voir, à contrôler les « missions extérieures » depuis un siège ghanéen, mais bien à former des responsables locaux, avec pour but «l'octroi de l'autonomie aux branches extérieures $^{3} \gg$. Dans un document plus récent, spécifiquement rédigé à cet effet, l'Eglise expose les contradictions de sa politique vis-à-vis des «branches extérieures »:

«L'objectif missionnaire clé de l'Église était d'établir et de nourrir les branches extérieures jusqu'à ce qu'elles grandissent et soient mûres pour pouvoir s'auto déterminer. Bien que l'Église ait clairement défini sa politique en ce qui concerne le mode d'opération, elle n'a rien dit à propos des conditions préalables pour l'accès à l'autonomie. Les branches externes ont joui du statut de régions/secteurs comme c'est le cas au Ghana pour faciliter l'administration ${ }^{4}$. »

En effet, dans le processus d'autonomisation d'une Église nationale, il n'est fixé « aucun calendrier ou échéance fixe compte tenu des différences prévalant dans chaque pays » (art. 6.1). Dans sa tentation universaliste, l'Église adopte une stratégie missionnaire qui a pour but l'évangélisation $\mathrm{du}$ monde, y compris du monde occidental. Cette ambition d'évangélisation «à l'envers » s'affirme tout particulièrement dans

\footnotetext{
${ }^{3}$ art. 16.1 (7) de la Constitution amendée de l'Église de Pentecôte, p. 51

${ }^{4}$ Rapport de la Commission sur l'autonomie des branches extérieures de l'Eglise de Pentecôte, document dactylographié, 1993, art. 5.2, p. 3
} 
l'unique cas connu de formation d'une assemblée «autochtone », en Ukraine, « composée principalement d'Ukrainiens », sous la direction d'un pasteur ghanéen. D'après le Président de l'Église, M. Ntumy, le missionnaire ghanéen, après avoir «implanté l'œuvre» en Ukraine et formé un responsable national «autochtone», devrait se retirer et laisser aux Ukrainiens une Église nationale autonome. L'Église de Pentecôte « ukrainienne » demeurerait cependant affiliée à la Church of Pentecost du Ghana, en tant que «branche » de celle-ci et serait gérée selon le modèle ghanéen. En effet, la Commission missionnaire sur l'autonomie des branches extérieures établit que «la Constitution des églises externes ou nationales existantes ou futures doit être calquée sur le modèle de l'Église de Pentecôte au Ghana et, lorsqu'elles auront obtenu l'autonomie, les principes énoncés dans la Constitution modèle s'appliqueront toujours ${ }^{5}$. » Mais ce type de configuration n'est pas fréquent, il est même plutôt exceptionnel et fait penser davantage à une expérimentation qui ferait de l'Ukraine un laboratoire d'essai de l'évangélisation «à l'envers». En effet, la Commission précise que «puisque la plupart des branches externes ont été initiées par des Ghanéens (...) il faudra qu'au moins soixante pour cent de la totalité des membres soient des résidents avant que le statut d'autonomie ne puisse être accordé à de telles églises, et ceci à la discrétion du Conseil Exécutif.» (art. 7.6, p. 7). Ainsi, à l'inverse de l'entreprise missionnaire coloniale qui a entrepris l'évangélisation des Noirs et la formation de pasteurs africains, l'Église ghanéenne, qui se pense comme l'instrument d'un plan divin qui ferait du Ghana une «nation missionnaire », se lance aujourd'hui dans l'évangélisation ou la «rechristianisation» des Blancs et la formation d'agents subordonnés. Plus concrètement, les rares cas de délégation des pouvoirs aux responsables nationaux «autochtones », comme c'est le cas au Burkina Faso, nous y reviendrons, ou en Côte-d'Ivoire et au Togo, ne se traduisent que par une autonomie relative des Églises nationales qui restent néanmoins étroitement liées et soumises à la direction ghanéenne qui continue de centraliser les ressources financières et le pouvoir décisionnel. En effet, le Conseil Exécutif International de la Church of Pentecost évalue les rapports annuels d'activités des missions et mouvements des Églises nationales, il est également «responsable des appels, des promotions, des nominations, des révocations et licenciements, de la mise en accusation, des affectations et de la retraite des ministres et des officiers de l'Église » ${ }^{\circ}$.

\footnotetext{
${ }^{5}$ Rapport de la Commission..., art. 7.5 relatif à la charte nationale, p. 6. De même l'Église autonome devra «conserver les dogmes, la doctrine biblique et les objectifs de l'Église de Pentecôte » (art. 9.3.1, p. 9).

${ }^{6}$ Constitution amendée..., art. $5.2(2)-$ g, p. 6.
} 
En Afrique de l'Ouest, seul le Burkina Faso semble échapper à l'expansion tentaculaire du siège ghanéen. Ce pays est l'une des toutes premières extensions de la Church of Pentecost du Ghana, puisque c'est à la fin des années cinquante (1959) qu'un groupe de pasteurs burkinabé négocie une affiliation au groupe de James McKeown. Parmi eux, le pasteur Pinnoaga Yanogo, d'origine mossi, qui sera le responsable national de l'Église de Pentecôte du Burkina Faso jusqu'à sa mort en 1988. Ce n'est qu'après cette date que deux responsables nationaux ghanéens prirent la direction de l'Église (de 1989 à $1996^{7}$ ), jusqu'à la nomination de l'actuel responsable national, également d'origine mossi. Ainsi, dans le cas du Burkina Faso, l'on peut dire que l'Église est restée, pour l'essentiel, aux mains des pasteurs mossi. L'absence de migration ghanéenne dans ce pays et la prise en main de l'Église par les pasteurs mossi fait de cette «mission» un cas d'exception qui, à la fois s'affranchit de la tutelle ghanéenne et reproduit localement le modèle dominant de l'exclusivité ethnonationale transposée en pays mossi ${ }^{8}$. Sur ce point, les pratiques de l'Église de Pentecôte du Burkina Faso sont semblables à celles décrites par P.-J. Laurent $(2001$; 2003) concernant les Assemblées de Dieu et la priorité donnée à l'évangélisation des Mossi ainsi que le recrutement exclusif de pasteurs Mossi. Une différence apparaît cependant puisque les pasteurs mossi, en tant «qu' auxiliaires privilégiés », ont pu être à leur tour missionnaires des Assemblées de Dieu en Afrique de l'Ouest, suivant les itinéraires de migration des populations burkinabé vers les pays côtiers, tandis que les pasteurs mossi de l'Église de Pentecôte ne contribuent pas à ce point à l'expansion missionnaire de leur Église. En effet, compte tenu de la centralisation du pouvoir à Accra, ce sont essentiellement les missionnaires ghanéens qui se déplacent.

Cette exclusivité ethnonationale mossi au sein d'une Église comme la Church of Pentecost pose aujourd'hui problème et il semble que les responsables ghanéens lui attribuent, au moins pour partie, le faible développement de l'Église dans ce pays. Depuis peu, des missionnaires ivoiriens sont mandatés au Burkina Faso pour seconder les pasteurs burkinabés et prendre en charge quelques assemblées jugées «endormies ». Les pasteurs mossi de l'Église de Pentecôte paraissent sur bien des points plus proches des «pasteurs-paysans » tels que les décrit P.J. Laurent (2003) et se démarquent assez nettement des «pasteursdocteurs », formés dans des Instituts bibliques internationaux et que l'on

\footnotetext{
${ }^{7}$ L'Apôtre B.K. Arthur de 1989 à 1992 et l'Apôtre J.K. Ampomah de 1992 à 1996, aux côtés desquels on note la présence de deux missionnaires togolais, Kokoe Mensah et Léon Agogo.

${ }^{8} \mathrm{Ce}$ thème a fait l'objet d'une étude antérieure sur « Les politiques identitaires d'une Église africaine transnationale », Cahiers d'Études Africaines, 172, 2003, pp. 857-881.
} 
rencontre plus souvent dans les pays côtiers, anglophones, comme le Ghana. Même si le responsable national de l'Église de Pentecôte du Burkina Faso fait état, outre un passage obligé par le Collège Biblique de la Church of Pentecost à Accra ${ }^{9}$, de formations dans des centres tels que le Haggaï Institute à Singapour (leadership), Corat Africa ${ }^{10}$ au Kenya (management), ou au Nigeria, ce n'est pas le cas de la plupart des pasteurs burkinabé de cette Église.

\section{Langue liturgique et identité ethno-nationale}

Si les fidèles burkinabé disposent d'une relative autonomie linguistique et liturgique vis à vis de l'administration ghanéenne, il n'en est pas de même pour les fidèles francophones en migration au Ghana. Au cours de nos missions successives à Accra, notre intérêt s'est porté tout spécialement sur les conditions d'existence des assemblées en français et en anglais au sein de la Church of Pentecost. C'est au début des années 1990 , en réaction au caractère « rural » et « indigène » des cultes en langue vernaculaire que se sont constituées trois assemblées en anglais : une à Koumassi, et deux à Accra (1993), dont l'une est installée dans le complexe PIWC (Pentecost International Worship Centre) dans le quartier de Kokomlemle à Accra. Une assemblée en français, créée en 1996, aujourd'hui dirigée par un Togolais (d'origine éwé), est ouverte aux fidèles francophones en migration au Ghana, principalement des Togolais, des Béninois et des Ivoiriens. Habituellement, les fidèles non Ashanti qui suivaient un culte en langue $t w i$ pouvaient espérer recevoir une traduction simultanée grâce à la présence d'un personnel bilingue. Cependant, cette pratique n'étant pas toujours respectée, les fidèles sont bientôt contraints d'apprendre la langue twi (parfois très éloignée de leur langue d'origine) ou de quitter l'assemblée. Le malaise progressif, les protestations des fidèles francophones et l'action militante de certains responsables ont abouti à la formation d'assemblées distinctes. Notre première interrogation a porté sur le statut de ces assemblées dont la formation marque nettement le refus de l'usage systématique de la langue twi comme langue de culte dans une Église qui associe étroitement l'usage de cette langue à son identité ethno-nationale. L'adoption de la langue nationale du Ghana,

\footnotetext{
${ }^{9}$ Ce qui impliqua, dans son cas, un apprentissage rapide de l'anglais par l'American Language Centre de Ouagadougou. L'Église de Pentecôte, en effet, met un point d'honneur à ne nommer que des pasteurs qu'elle a elle-même formés, ce qui implique leur passage par l'incontournable Pentecost Bible Training Institute (BTI) de l'Église.

10 "Kenya Pan-African management courses for churches and church related organisations" www.oscar.org.uk
} 
l'anglais, ou de la langue française lorsqu'il s'agit de migrants francophones, témoigne dans les deux cas d'une volonté affichée et délibérée de ne pas adhérer au modèle d'identification communautaire (voire ethnonational, puisqu'il est essentiellement véhiculé par l'usage de la langue vernaculaire) proposé par une Église qui demeure profondément attachée à son ancrage «indigène ». L'assemblée en français quant à elle voit son action se doubler d'une fonction sociale. En effet, l'intégration à une communauté de croyants francophones constitue un refuge sécurisant face aux difficultés d'intégration dans un pays anglophone. De ce point de vue, cette assemblée en particulier est perçue par les dirigeants les plus conservateurs comme l'affirmation d'un particularisme identitaire. Les fidèles de cette assemblée sont le plus souvent des étudiants venus du Bénin, du Togo ou de la Côte-d'Ivoire dans le but d'apprendre l'anglais ou d'effectuer au Ghana une formation plus cotée que dans leur pays d'origine. Il s'agit plus souvent de formations techniques, mais pour certains, la fréquentation de l'université suffit à favoriser la rencontre avec les étudiants pentecôtistes qui ne tardent pas à attirer ces jeunes migrants désorientés vers une assemblée où ils seront pris en charge.

La formation de ces assemblées au sein d'une Église qui se pense « indigène » est l'objet d'une controverse qui divise jusqu'à la classe dirigeante. Au sommet, le Président de l'Église Internationale, l'Apôtre Michael Ntumy, qui par son discours pour le moins ambivalent, cristallise à lui seul les tensions liées à la «politique identitaire» de l'Église. L'élection de ce jeune docteur en philosophie religieuse et en théologie, parlant couramment la langue française ${ }^{11}$, auteur de plusieurs ouvrages en français et en anglais, vient rompre avec l'héritage des «anciens », les « patriarches » qui l'ont précédé à la direction de l'Église. Parallèlement à son ambition de fonder une «Église noire », le «missionnaire-fondateur » lui-même, James McKeown, refusa pourtant jusqu'à son retrait en 1982 la nomination d'un Président ghanéen ${ }^{12}$. La rupture se fait donc à un double niveau : celui de l'âge du Président (Michael Ntumy avait quarante ans lorsqu'il fut élu en 1998 et son mandat est limité) et le niveau de qualifications (contrairement au missionnaire-fondateur, qui ne fut pas scolarisé au-delà de l'âge de 11 ans (Leonard, 1989), le jeune Président,

\footnotetext{
${ }^{11}$ De ce point de vue également, le Président fait partie des rares dirigeants qui ont mis à profit leur expérience de missionnaire pour apprendre la langue des pays où ils étaient affectés : il apprit le français lorsqu'il était missionnaire puis responsable national de l'Église de Pentecôte de Côte-d'Ivoire (1991-1996) puis en France (1996-1998).

${ }^{12}$ A la suite de ce long règne, l'Église opta pour des mandats d'une durée de cinq ans, renouvelables une seule fois, afin de permettre une passation régulière du pouvoir, ce qui explique que deux Présidents se soient succédés, le Rév. F. S. Safo (1982-1987) et le Prophète M. K. Yeboah (1988-1998: deux mandats), depuis le retrait de James McKeown à l'actuel Président (1998), dont le mandat a été renouvelé en 2003.
} 
comme d'autres dirigeants de plus en plus nombreux, s'est engagé dans des cycles d'études doctorales en Europe et au Canada). L'élection de cette nouvelle figure de «pasteur-docteur » à la tête d'une Église telle que la Church of Pentecost, n'a pas eu d'évidence au départ et a rencontré de très fortes résistances. Elle témoigne aujourd'hui d'une volonté de donner à l'Église une allure «moderniste» qui s'inspire du mouvement néopentecôtiste et se veut moins populaire, plus «intellectualiste », dans une certaine mesure. La formation d'assemblées en français et en anglais était un premier pas dans ce sens, une revendication «progressiste » qui émergeait de la base (les étudiants pentecôtistes), au moins dans un premier temps, mais qui fut bien vite soutenue par quelques dirigeants. L'initiative, cependant, ne fit pas l'unanimité, y compris au sein de la direction, et les pressions exercées pour limiter le phénomène, ou le contenir, sont parfois assez fortes.

Dans l'entretien que nous a accordé le Président Michael K. Ntumy (2003), celui-ci se place immédiatement en défenseur de ces assemblées qui sont pour lui un gage d'ouverture de l'Église à la «communauté internationale ». Il rappelle qu'il fut lui-même missionnaire et président de l'Église en Côte-d'Ivoire et qu'il avait soutenu l'adoption progressive de la langue française dans ce pays. La première « assemblée en français » avait été créée à l'initiative d'un Ivoirien, l'Ancien Robert Mensah, en 1988, alors que l'Apôtre togolais B. Y. Apedo était président de l'Église et avec son soutien. Devant le nombre croissant de fidèles ivoiriens dans les assemblées, il avait encouragé les responsables ivoiriens à opter ponctuellement pour l'usage du français, «lorsque les francophones étaient plus nombreux que les Ghanéens », précise l'Ancien Mensah", puis à former des groupes de fidèles francophones. Cette initiative, même dans un pays francophone, ne fit pas l'unanimité parmi les missionnaires ghanéens qui craignaient une division au sein de l'Église sur la base d'une revendication identitaire «francophone ». Rappelons que c'est exactement ce qui s'est produit au début des années 1980 lorsqu'un groupe dissident, mené par un fidèle du nom de Yao Bio, s'opposa à la «présence ghanéenne » en revendiquant son identité ivoirienne à travers l'usage de la langue française, s'inscrivant ainsi dans une démarche nationaliste. $\mathrm{La}$ langue française était devenue l'instrument de la contestation et de l'affirmation identitaire et nationaliste. C'est pourquoi, devant la création d'une assemblée en français, la direction ghanéenne craignait les conflits potentiels entre fidèles ghanéens et ivoiriens francophones et par suite, la rupture de ces assemblées avec l'Église. Rapidement, en effet, la minorité de fidèles francophones s'est vue victime d'une stigmatisation agressive de

\footnotetext{
* Entretien réalisé par courrier électronique en novembre 2003, la crise ivoirienne ne permettant pas de se rendre dans le pays.
} 
la part d'une communauté de fidèles majoritairement ghanéenne. Mais l'accord fut donné par le Président ghanéen M. K. Yeboah qui adressa une note à l'Ancien Mensah lui accordant l'ouverture officielle d'une «Section en Français » ${ }^{13}$ aujourd'hui installée à Treichville. Lorsqu'en 1991, Michael Ntumy fut affecté à la tête de l'Église de Pentecôte de Côted'Ivoire, il choisit de soutenir cette politique et d'en faire une stratégie de conversion des «francophones ». Plusieurs autres assemblées en français furent créées à la suite de la «Section en Français » de Treichville tandis que les fidèles ghanéens se regroupèrent dans des assemblées distinctes comme c'est également le cas à Ouagadougou, au Burkina Faso. La nomination du premier Apôtre ivoirien, Sidiki Traoré ${ }^{14}$, en 1996 permit de banaliser et de généraliser l'usage du français dans les assemblées ivoiriennes au point que «le twi a commencé à disparaître, déclare un pasteur ivoirien en 2003. La plupart des assemblées aujourd'hui font le culte en français ».

Mais les rivalités entre fidèles francophones et ghanéens ne sont pas totalement dépassées par ces évolutions. Elles paraissent au contraire si structurelles qu'elles dépassent largement le contexte national de la Côted'Ivoire. Selon le Président Ntumy, la tension des relations entre les Ghanéens et les Ivoiriens, ou les «francophones » en général, s'explique par un rapport de concurrence, où se mêleraient à la fois une admiration non avouée et la contestation de l'hégémonie ghanéenne au sein de l'Église. L'Église étant la «création» des Ghanéens ${ }^{15}$, et les pasteurs ghanéens étant considérés comme «historiquement» et (donc) « spirituellement» plus «avancés » dans «l'œuvre », les missionnaires ghanéens en Côte-d'Ivoire sont perçus comme des émissaires qui profitent de leur mission pour "occuper le terrain » massivement et imposer leur vision. Le Président évoque, à juste titre, la crainte de la «colonisation » ghanéenne et ses termes nous renvoient aux principaux arguments évoqués par Yao Bio, qui portaient précisément sur les modes «d'occupation » et de «gestion» des assemblées par les dirigeants ghanéens (Fancello, $2003 \mathrm{~b})$. Au regard de la chronologie dans laquelle prend place la création des «assemblées en français » en Côte-d'Ivoire (nous sommes au début

${ }^{13}$ L'expression «Section en Français » serait tirée de la note du Président ainsi rédigée : "You are allowed to open a French Section of the Church in Abidjan 》 de laquelle s'inspira l'Ancien pour nommer la nouvelle assemblée.

${ }^{14}$ Qui est actuellement le représentant des pays francophones au Conseil Exécutif International de la Church of Pentecost.

${ }^{15} \mathrm{La}$ figure du «fondateur», le missionnaire James McKeown, étant bien souvent effacée derrière la «prise en main » de l'Église par les pasteurs ghanéens, d'abord en tant qu'associés, puis sans partage. L'hommage à la mémoire du fondateur fait l'objet de « journées McKeown », au cours d'une semaine missionnaire organisée chaque année au mois de mars. 
des années 1990 - la dissidence nationaliste ivoirienne est déjà largement opérée et constitue l'événement le plus marquant de l'histoire récente de l'Église dans ce pays), l'initiative de l'Ancien Mensah et l'accord du Président M. K. Yeboah peuvent être perçus comme une volonté de contrecarrer le potentiel déstabilisateur de la dissidence ivoirienne, de même que la politique d'ouverture menée par le président Ntumy à leur suite sonne comme une réponse directe aux arguments du leader séparatiste qui, par le conflit qu'il a provoqué, a déclenché ce mouvement de restructuration interne de l'Église ghanéenne.

Du point de vue du siège ghanéen, il s'agit pourtant moins d'une restructuration profonde de l'Église que d'une concession faite aux minorités francophones afin d'éviter une hémorragie de fidèles vers le groupe dissident. Même si une partie des dirigeants, tout comme le Président, déplore aujourd'hui certains traits « indigènes » de la Church of Pentecost et le manque d'ouverture de ses membres, la plupart restent attachés à leur identité originelle et perçoivent l'Église comme le «fleuron » des Ashanti, au Ghana comme à l'étranger. Dans son mémoire de maîtrise de théologie (2000), le Président M. Ntumy identifie plusieurs

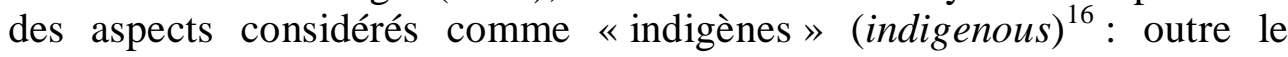
rapport à la musique et à la danse (traditional drumming and dancing), il cite notamment l'usage de la langue vernaculaire, le twi, la division spatiale des sexes dans les assemblées (sitting arrangement in church) et l'obligation pour les femmes de se couvrir la tête (head covering) (p. 35). Il justifie ces traits par le choix d'une politique d'indigénisation de l'Église qui fut le moteur, selon lui, de son ascension fulgurante au Ghana. Certaines de ces normes disparaissent dans les nouvelles assemblées en français et en anglais.

Cette perception de l'Église comme profondément ghanéenne, c'est-àdire faisant du Ghana une "nation missionnaire» et de la Church of Pentecost une "Eglise nationale », est essentiellement véhiculée par une base qui constitue l'essentiel des effectifs de fidèles et de dirigeants. En effet, comme l'indique par ailleurs Paul Gifford (1998: 76), l'Église de Pentecôte au Ghana s'appuie sur une base numérique importante d'Anciens (elders) placés sous l'autorité d'un pasteur de district. L'Église ainsi conçue selon une structure pyramidale du pouvoir, oppose à une large base « indigène » une élite de dirigeants, incarnés notamment par les nouveaux «pasteurs-docteurs », comme M. K. Ntumy, qui véhiculent, pour certains et certains seulement, une vision plus «progressiste » et ouverte de l'Église. Cette vision se traduit par une politique ambivalente

\footnotetext{
${ }^{16}$ Notons à cette occasion que le terme « indigène » est utilisé par les acteurs euxmêmes, fidèles et pasteurs de la Church of Pentecost, de même qu'il apparaît dans la littérature de l’Église.
} 
qui d'une part reste fidèle au choix premier de la vernacularisation et d'autre part ménage l'adoption de mesures qui contrastent avec le caractère indigène de l'Église, comme le recours aux langues nationales dans les assemblées en français et en anglais, ou la libéralisation de certaines normes vestimentaires pour les fidèles de ces assemblées. Cette ambivalence alimente une série de tensions liées aux contradictions de la «politique identitaire» de l'Église dans la rencontre avec les autres communautés africaines. Une partie de la direction ghanéenne continue de voir dans la formation de ces assemblées «réformistes» un danger de division au sein d'une communauté de «frères et sœurs en Christ » jusque là unie autour d'une identité unique. Ainsi, la pluralité identitaire qui naît de l'expansion missionnaire est autant redoutée sur le plan pratique qu'elle est affichée sur le plan politique.

\section{La « francophonie », une identité réactive?}

A Accra, l'Ancien responsable de l'Assemblée en français de Kokomlemle se plaint régulièrement des pressions exercées par l'Église pour l'intégration des fidèles ghanéens dans cette assemblée. De même, l'affectation d'un pasteur ghanéen dans cette assemblée, au cours de l'année 2002, fut perçue comme une volonté de contrôle de l'assemblée francophone. Ce pasteur, en tant que responsable de l'assemblée, est normalement amené à prêcher régulièrement. Mais sa maîtrise de la langue française ne lui permettant pas de prêcher en français, il a recours le plus souvent à l'anglais et l'assemblée se voit contrainte de suivre un culte traduit en français. On comprend alors que pour les fidèles de cette assemblée, autant que pour l'Ancien à l'origine de sa création, l'affectation de ce nouveau pasteur est un coup porté au (ré)confort que leur procurait le culte en français. Pour certains d'entre eux qui se sont convertis à leur arrivée au Ghana, l'intégration dans une communauté francophone a probablement été un élément déterminant de leur démarche. Pour d'autres, déjà convertis et qui fréquentaient l'Église de Pentecôte dans leur pays d'origine (le Bénin et la Côte-d'Ivoire sont des pays où l'Église est relativement bien implantée), la constitution de cette assemblée en français représente le plus sûr moyen pour eux de poursuivre la fréquentation de cette Église à l'étranger, tout en leur procurant le réconfort intérieur de se sentir toujours au sein du même réseau d'Église. L'implantation de l'Église de Pentecôte est toujours si étroitement liée à la migration de ses fidèles qu'elle met un point d'honneur à paraître comme un repère en Afrique et en Europe, notamment en préservant toujours la même dénomination. Mais ce privilège semble davantage réservé aux 
Ghanéens à l'étranger qu'aux fidèles francophones alors même qu'ils proviennent de pays voisins où l'Église a de nombreuses assemblées. Les entretiens menés auprès de ces fidèles révèlent que ceux qui étaient déjà membres de l'Église de Pentecôte dans leur pays, vivent assez mal ce qu'ils dénoncent comme un manque de considération de la part de la communauté ghanéenne à leur égard et le mépris que leur attirent leurs revendications, perçues comme l'expression d'un repli identitaire. En effet, le pasteur ghanéen affecté dans cette assemblée, loin de ménager ces « espaces identitaires », y réintroduit la langue anglaise d'abord, puis progressivement la langue twi et réaffirme ainsi le primat de l'identité ghanéenne de cette Église. Le Président Ntumy justifie cette affectation par le manque de personnel francophone (qui est lui même par ailleurs le résultat du refus par les dirigeants ghanéens d'une délégation des pouvoirs à des responsables francophones).

L'usage de l'anglais revêt un caractère particulier. Cédric Mayrargue l'associe à un pouvoir d'attraction supérieur à celui de la langue française qui aboutit, au Bénin, à un usage de plus en plus répandu de l'anglais par les prédicateurs en dépit du fait que le Bénin est un pays francophone (2001 : 286). Cette situation rappelle singulièrement les conditions du passage à Ouagadougou (Burkina Faso) d'une croisade d'évangélisation organisée par la Church of Pentecost du Ghana en 1999. Les prédicateurs ghanéens avaient programmé trois jours et trois nuits d'activités quasi ininterrompues durant lesquels ils eurent exclusivement recours à l'anglais. Quelques interprètes improvisés reçurent pour tâche de donner une traduction simultanée des prédications, en français et en möré, mais la diction du prêche pentecôtiste laisse si peu de possibilités à un traducteur, tant elle se caractérise par de multiples accélérations et répétitions, que le recours à la (double) traduction, autant que le recours exclusif à l'anglais, furent perçus par les auditeurs comme inappropriés. En effet, l'excitation et la diction effrénée de l'orateur obligent souvent les traducteurs à réduire considérablement le contenu de leur traduction et donc du prêche, ce qui en réduit inévitablement la portée. Ce type de mise en scène est dénoncé aujourd'hui par une minorité de dirigeants qui considère ces pratiques inefficaces et inappropriées pour l'évangélisation des pays francophones. Parallèlement, les Églises nationales des pays francophones se plaignent de plus en plus souvent de la présence de dirigeants ghanéens qui ne parlent pas le français. Outre les conflits et dissidences connus en Côted'Ivoire, au Togo et au Bénin, des pays comme le Sénégal ou encore la France, voient leur communauté de fidèles se diviser autour de ces questions qui illustrent les aspects autoritaires de cette Église.

Plus récemment à Accra, nous avons pu assister à une scène de résistance manifeste de la part de l'Ancien (togolais) en charge de «l'assemblée en français» de Kokomlemle. En l'absence du pasteur 
principal, il s'est vu appelé à prêcher, comme il en avait l'habitude bien avant l'affectation du pasteur. Ce jour-là, durant les premières séquences de culte (adoration, prières et chants), le diacre chargé de diriger ces séquences s'était jusque là exprimé en français, mais avait dû accepter bien malgré lui d'être simultanément traduit en anglais. Cette situation, à laquelle les fidèles francophones étaient peu habitués, eut pour effet de provoquer sinon l'exaspération, au moins le désintérêt progressif de certains d'entre eux pour les interventions du diacre. Le respect dû par les fidèles à l'un de leurs responsables ne leur permettait pas cependant de manifester ouvertement leur sentiment, mais leurs visages en disaient long sur leur mécontentement. Ainsi, le moment venu où l'Ancien prit la parole pour la prédication, la tension était montée parmi les fidèles. L'Ancien, qui dit avoir relevé depuis plusieurs mois un manque d'intérêt croissant chez ses fidèles, attribue ce résultat à la formation progressive d'un culte bilingue malgré le fait que les fidèles de cette assemblée soient exclusivement francophones. Ce jour-là, prenant place, il salua l'assemblée et la remercia de son attention, tandis que la traductrice, une jeune étudiante ghanéenne, traduisait ses premiers mots. Bientôt gêné par «l'écho» de la traduction, et irrité par l'absurdité de la situation, il marqua un temps de silence, comme pour s'assurer de sa propre détermination, puis, dans un climat de tension déjà extrême, le visage serré, il s'élança dans une manœuvre adroite mais risquée, cherchant à ménager les responsables présents (d'autres Anciens, également ghanéens). S'adressant à «son» assemblée, il demanda d'une voix adoucie : «Y a-t-il des personnes parmi vous qui ne comprennent pas le français ? ». La formule fut accueillie avec un certain trouble de la part des fidèles, tous francophones, appelés à la résistance et surpris par l'audace de leur responsable. Tous répondirent par la négative. Un nouveau temps de silence mit la scène en suspens, avant que la traductrice ne reprenne la parole et rappelle la présence d'un fidèle ghanéen non francophone pour qui la traduction s'avérait nécessaire. L'assemblée suivait le mouvement avec une attention soutenue. L'Ancien, sans perdre son sang froid, se tourna vers la traductrice et l'invita à faire la traduction auprès du fidèle, en s'installant à ses côtés, de manière à ce que le culte puisse être dirigé en français, pour une assemblée qui s'affirmait une fois de plus, totalement « francophone ». L'instant d'après, en suivant le regard latéral de l'Ancien en direction de ses collaborateurs, tandis que la traductrice allait s'installer auprès du fidèle ghanéen, nous avons pu relever sur les visages des responsables ghanéens les traits de la stupéfaction devant ce qu'ils considéraient certainement comme un affront direct à leur égard, et un coup bas en l'absence du pasteur principal. L'Ancien, cependant, qui nous fit part de son exaltation à la sortie du culte, savourait sa victoire, bien que ponctuelle, tout en déplorant une fois de plus les tentatives manifestes des 
dirigeants ghanéens pour réintroduire la langue anglaise dans cette assemblée francophone, voire le twi, par le biais de quelques cantiques « levés » spontanément par l'un d'entre eux, ce qui a pour effet de laisser les fidèles francophones dans une perplexité qu'ils ont souvent bien du mal à dissimuler.

Nous avons pu observer des situations similaires à plusieurs reprises, dans d'autres lieux (Paris, Bruxelles), et les entretiens menés auprès de ces fidèles et de leurs responsables attestent d'un malaise collectif, symptomatique d'une crise politique profonde dans les relations de l'Église avec les autres communautés africaines. Si la formation d'une communauté francophone au sein de l'Église constitue une sorte de « niche communautaire », en réalité, cette «francophonie » se construit plutôt comme une identité réactive face à l'impérialisme ghanéen, c'est-àdire que ces acteurs sont d'autant plus conduits à s'affirmer en tant que «francophones » que les Ghanéens leur opposent un modèle impérialiste et dominateur. Ainsi, la question du choix de la langue liturgique, qui pourrait paraître un détail dans l'organisation de l'Église, révèle en fait de fortes tensions qui agitent l'Église depuis la base, la communauté de fidèles, à la classe dirigeante. En effet, une partie de la direction de l'Église déplore le cloisonnement des communautés de fidèles, notamment à l'étranger, et le fait que les responsables dans les «missions extérieures », notamment en Europe, puissent demeurer plusieurs années dans un pays sans en apprendre la langue nationale. Certains pasteurs affectés plusieurs années en France, en Belgique ou en Italie n'y ont pas appris les moindres rudiments de français ou d'italien. Ce fait souligne le caractère étroitement communautaire de la diaspora ghanéenne. Les assemblées, ainsi composées de migrants (Ashanti), usant exclusivement de la langue twi comme langue de culte, sont considérées par certains responsables comme trop «enfermées sur elles-mêmes ». $\mathrm{Ce}$ cloisonnement communautaire contribue à limiter de beaucoup le recrutement de nouveaux fidèles parmi les autres communautés africaines.

Le recours à la traduction qui précédait la création d'assemblées distinctes (en français et en anglais) a permis par ailleurs, il faut le rappeler, l'émergence d'une catégorie d'agents, à la fois proches de la classe dirigeante et des assemblées: les interprètes. Leurs parcours de conversion, leur situation familiale et leur formation universitaire les placent d'emblée dans une perspective transnationale, par la migration de leurs parents (souvent entre le Ghana et la Côte-d'Ivoire, le Bénin ou le Togo), la «double nationalité », officielle ou symbolique, qui en résulte parfois et leur trajectoire personnelle. Au sein de l'Église, leur formation a permis à plusieurs d'entre eux d'accéder à des emplois salariés parmi les « départements » qui la composent, le plus souvent comme «secrétaire » ou traducteur officiel. Pour d'autres, l'ambiguïté de leur (double) statut, 
parfois diacre ou ancien en même temps que secrétaire ou responsable d'une assemblée locale, peut perdurer plusieurs années avant de se traduire par l'accès à un poste de pasteur à plein temps (full-time ministry). Considérés par ailleurs comme des «intellectuels », ils suscitent parfois la méfiance de la direction ${ }^{17}$ d'autant plus qu'ils sont reliés entre eux et à quelques dirigeants par un réseau informel et parallèle qui forme un consensus autour de l'adoption des langues nationales, ou du soutien à la promotion de dirigeants «autochtones » à la tête des missions extérieures encore dirigées par des pasteurs Ghanéens. Certains traits considérés « indigènes », comme la division sexuelle au sein du temple ou le port du foulard pour les femmes, sont discutés par ces agents. Les interprètes sont parmi les agents les plus hybrides de la hiérarchie de cette Église car ils sont eux-mêmes le produit historique de la confrontation des missionnaires ghanéens aux autres communautés ouest-africaines, principalement francophones. Tout comme les leaders de camps de prière, leurs fonctions les rendent à la fois ambigus et incontournables au sein d'une Église ébranlée dans ses postures les plus fermes.

\section{Les camps de prière (prayer camps) et la délivrance}

Parmi les sujets de controverse et les espaces d'ambivalence au sein de la Church of Pentecost, la question épineuse du statut des camps de prière (prayer camps) dans la structure de l'Église est depuis longtemps au centre d'un débat qui traverse de nombreuses Églises pentecôtistes. Pour Paul Gifford (1998), la Church of Pentecost est l'une des Églises les plus étroitement associées à ce débat, compte tenu des nombreux camps qui exercent sous sa dénomination et des nombreuses dissidences qui sont associées à son histoire. Les contradictions relatives au statut des camps de prière et de leurs dirigeants sont particulièrement représentatives de la posture ambiguë de l'Eglise de Pentecôte vis-à-vis de ces «structures » à part. L'histoire de ces camps est complexe et mouvementée. Bien que l'apparition des camps de guérison soit très tôt associée à l'Église de Pentecôte, ce n'est qu'au début des années 1970 qu'ils sont reconnus comme faisant partie intégrante des activités de l'Église. D’abord créés sous la forme de healing post à la charge d'un leader, ils sont aujourd'hui

\footnotetext{
17 Les éléments recueillis depuis plusieurs années, aussi bien auprès du siège de l'Église qu'à l'extérieur du Ghana, nous permettent d'attester qu'il y aurait bien eu une politique de promotion des «intellectuels » au sein de la Church of Pentecost durant ces dernières années. Cependant, depuis peu, les tensions et les contradictions liées à ce choix politique, qui se sont parfois traduites par des dissidences réelles, ont suscité une certaine frilosité au sein du Conseil Exécutif quant au recrutement ou à la promotion « d'intellectuels ».
} 
constitués en véritables institutions au sein même de l'Église et dirigés par des leaders «charismatiques» que l'Église peine à soumettre à son autorité. Les «leaders» des camps sont souvent des diacres (ou des diaconesses) qui s'autoproclament «prophète/prophétesse » sans formation religieuse, au sens de ce qui «qualifie », pour la direction, c'està-dire le passage par l'Institut Biblique de formation et l'avancement contrôlé dans la hiérarchie de l'Église. L'expansion transnationale de ces camps semble se calquer sur celle de l'Église puisque de nombreux fidèles (particulièrement ivoiriens et togolais) se rendent au Ghana pour $y$ séjourner, et que des camps s'implantent en Côte-d'Ivoire et au Togo, le plus souvent sous la direction de leaders ghanéens. Les camps se présentent ainsi comme des lieux de passage de migrants guidés par la seule renommée d'un «prophète-guérisseur» ou d'une prophétesse, lesquels contribuent à tisser un véritable réseau transnational de «prophètes-guérisseurs ». Pour contrecarrer le succès des camps de prière et de leurs leaders, la direction de l'Église tente de réintroduire la guérison miraculeuse et la délivrance au sein des cultes hebdomadaires des assemblées de quartier, sous la direction et l'autorité d'un pasteur. Ces camps sont en effet très nombreux et le débat est virulent dans les pays côtiers comme le Ghana et la Côte-d'Ivoire.

Dès les premières années (1954), le débat suscité par l'apparition des camps de prière porte sur le statut des dirigeants de ces camps et sur leur rapport à l'autorité de l'Église, c'est-à-dire, à leur contrôle. Comme l'indique E. K. Larbi, "the activities at these centres almost exclusively gravitate around one key person: a prophet, a prophetess, or an evangelist ${ }^{18}{ }$. Le mode de fonctionnement du premier d'entre eux, dirigé par G. A. Lawson, membre de la Church of Pentecost, que Larbi considère comme le précurseur des camps de prière, soulève d'emblée une controverse d'ordre liturgique à propos de l'utilisation par Lawson d'une « eau bénite» (blessed water) et de «mouchoirs bénits» (blessed handkerchiefs). Outre le rapport à l'orthodoxie de la jeune Église, ces innovations liturgiques traduisent déjà le caractère indépendant du leader vis à vis de son Église au point que Larbi souligne : "It would be difficult for an outsider to see it as part of McKeown's organisation » (2001:371). Le conflit qui s'en suit aboutit bientôt à la séparation du camp et de l'Église (1958). Le camp devient à son tour une Église ${ }^{19}$ et vient allonger la longue liste des dissidences qui jalonnent l'histoire de la Church of

\footnotetext{
${ }^{18}$ Larbi, 2001 : 367. Le titre de «prophète» ou «prophétesse » est bien plus souvent attribué aux leaders des camps qu'il n'est en fait reconnu par l'Église, sachant que le statut «officiel» ou «ecclésial » de Prophète, au sein de cette Église, est lui-même ambigu.

19 Après la dissidence de 1958, le Divine Healing Post devient la Divine Healer's Church (Larbi, 2001).
} 
Pentecost. Au regard de l'histoire récente et des débats qui travaillent cette Église encore aujourd'hui, le cas du premier healing post se présente comme une sorte de scénario paradigmatique qui va se répéter dans des termes similaires, au Ghana bien sur (voir Fancello, 2003a), mais également dans d'autres pays, comme la Côte d'Ivoire, où apparaissent bientôt les premiers camps de guérison, calqués sur le modèle ghanéen. Les dissidents eux-même, comme Yao Bio en Côte-d'Ivoire, n'en reproduisent pas moins des camps inspirés de ceux de l'Église ghanéenne. Les camps que nous avons pu visiter, celui de Yao Bio situé dans le quartier de Williamsville à Abidjan, de «maman Haggar » à Yopougon ou celui de «Sister Kate» à Ablekuma (Accra), sont au centre d'une polémique qui met en cause le statut de ces leaders et de leur activité. Yao Bio, depuis sa dissidence avec l'Église de Pentecôte, est passé du statut de simple fidèle à celui de leader de son propre camp (il est déjà président de sa propre Église), ce qui lui pose un problème de légitimité, tandis que l'autorité de "maman Haggar », une ghanéenne installée en Côte-d'Ivoire, est contestée par ses propres collaborateurs. De son côté, l'Église multiplie les contrôles dans les camps afin de toujours contenir les tentations indépendantistes des leaders et de vérifier la conformité de leur activité avec l'orthodoxie de l'Église.

Les camps de prière sont le plus souvent situés dans les quartiers périphériques des villes (Yopougon), voire en milieu rural (Ablekuma). Sur le plan thérapeutique, les guérisons sont plus souvent présentées comme des guérisons de la «folie », d'infirmités ou de malformations physiques attribuées à l'entremise de démons ou esprits malfaisants, ou de « dérangements sexuels » attribués aux maris/femmes de nuits, des esprits qui visitent leurs victimes dans leur sommeil et simulent avec elles des actes sexuels. Dans le même registre, la masturbation est également attribuée à des esprits malfaisants, ce qui fait dire à certains fidèles qu'ils sont « victimes de l'esprit de masturbation ». La structure du camp, à la différence d'un temple de quartier, a essentiellement pour fonction de permettre la résidence sur place et l'encadrement des malades. Les personnes considérées comme dangereuses ou violentes (principalement celles que l'on considère atteintes de «folie ») sont cloîtrées dans l'un des bâtiments constitué de «box» disposés de part et d'autres d'un couloir central et d'où proviennent les cris et gémissements des personnes enfermées et enchaînées (Yopougon). Ces cris et gémissements, loin d'être interprétés comme des cris de détresse, voire de douleurs (enchaînement, immobilité, incommodité) sont attribués à l'agitation des démons responsables de la folie. Certains parmi les «malades » qui connaissent des accalmies au cours de leur séjour peuvent être libérés durant la journée et l'on peut ainsi voir des personnes déambuler dans le camp (Ablekuma), portant à la main une chaîne métallique dont l'extrémité est reliée à l'un de 
leurs pieds, de manière à ce qu' elles puissent être rattachées à la tombée de la nuit, par «mesure de sécurité » pour les autres malades. Les personnes résidentes sont rassemblées dans le temple, où sont disposés des matelas de mousse à même le sol ou, dans le meilleur des cas, des sommiers de bois. Certaines familles, accompagnant le «malade», construisent des petits baraquements dans l'enceinte du camp dont le temple est le centre de gravité (Yopougon). Les résidents disposent en général de peu d'effets personnels et l'isolement du camp réduit leurs déplacements aux alentours du temple (aucun ne s'approche de la résidence du dirigeant, laquelle est soigneusement disposée à l'écart et quelques fois même, cloisonnée). Certains dorment à même les bancs du temple, des hommes le plus souvent. Hommes et femmes sont évidemment soigneusement séparés, installés dans deux halls différents ou dans des espaces clairement délimités. Les journées au camp sont organisées autour des cultes, plusieurs par jour, en général un le matin, un autre à la mi journée et un dernier le soir. Dans les camps « associés » à l'Église, celle-ci impose que soit dispensé un «enseignement», conformément à la structure du culte pentecôtiste, c'est-à-dire une prédication basée sur une lecture de la Bible, précédant la séance de prière pour la «guérison des malades». Cette mesure a pour but de corriger autant que possible l'aspect clientéliste des camps de prière.

Les camps offrent, en effet, une large panoplie de services qui dépassent largement le caractère thérapeutique de leur activité. Selon les données recueillies par Rijk van Dijk, les camps peuvent également jouer le rôle de structures de transit pour les migrants ghanéens en attente de leurs « papiers », passeport ou visa (1997 : 145), voire de plaque tournante pour les voyageurs à travers l'Afrique de l'Ouest ou vers l'Europe et les Etats-Unis. Les difficultés administratives, au même titre que tout autre «problème » de la vie quotidienne, étant assimilées à des «blocages » et attribuées aux mauvais esprits, le traitement par la prière, que l'on dit ici, «d'intercession », se présente comme un recours pratique pour «briser » ou « défaire » le mauvais sort qui pèse sur les projets de migration. Par ailleurs, la structure des camps étant peu répandue en dehors du Ghana, les Ghanéens installés en Europe n'hésitent pas à retourner «au pays », pour des raisons de santé, lorsque la médecine occidentale n'a pu les guérir. L'extrême variété des cas traités dans ces camps et la relation des fidèles à leur leader, contrastent avec les séances de délivrance collective que l'on peut observer dans les temples de quartier où la relation du pasteur à ses fidèles est bien moins individualisée et ne laisse place à aucune prise de parole de la part des fidèles. Mais les analyses de R. van Dijk (1997) vont plus loin en suggérant que les leaders de camps disposent d'une popularité internationale au sein du réseau de migration ghanéenne intercontinentale. C'est le décalage entre les deux modes de fonctionnement, la dimension 
charismatique des leaders opposée au «charisme de fonction» des pasteurs officiels et la plus grande adaptabilité des traitements offerts par les camps qui suscitent la méfiance et la volonté de contrôle de la part de la direction de l'Église.

Pour l'Église, la seule différence que peuvent laisser paraître les «cultes » tels qu'ils sont organisés dans les camps, par rapport à ceux que l'on peut observer dans les assemblées de quartier, est l'ajout d'une séquence de prière pour la guérison des malades. Pour le reste, le culte est supposé être dirigé dans la stricte fidélité à l'orthodoxie de l'Église de Pentecôte. Les camps de prière sont officiellement associés à l'Église, à la condition d'en accepter les principes (politiques), ce qui se traduit d'abord par la mise sous tutelle du camp, placé alors sous l'autorité d'un pasteur de district au même titre qu'une assemblée locale. Dans la réalité, les responsables des camps de prière étant peu formés à la direction des cultes, la partie réservée à «l'enseignement », c'est-à-dire la prédication, se trouve extrêmement réduite au profit de la séance de délivrance individuelle et collective qui mobilise bien plus l'attention des fidèles dans l'attente de leur guérison miraculeuse. La plupart des camps sont dirigés par des personnes qui n'ont pas le statut de « pasteur» au sein de l'Église, ni la formation associée à ce titre, mais qui se sont révélées par des dons (de guérison, de vision ou de discernement des esprits et de prophétie) et qui font usage de ces dons au cours de séances de délivrance destinées à des personnes venues spécialement chercher une guérison. Ainsi, les responsables de camps ne sont pas rémunérés par l'Église. Le camp tire l'essentiel des revenus nécessaires à son fonctionnement des dîmes et offrandes des résidents (ou de leur famille), de dons de personnes guéries dans le camp (sous la forme de matériel de construction, le plus souvent), éventuellement d'une aide substantielle et ponctuelle de la part de l'Église. La priorité donnée à la quête de guérison donne à la délivrance une dimension clientéliste qui pervertie, aux yeux de l'Église, le rôle des responsables de camps. Ces derniers passent pour des personnalités « charismatiques » dont la réputation internationale est bien supérieure à celle des pasteurs et Apôtres de l'Église. La perception des fidèles en quête de guérison, loin d'intégrer la hiérarchie propre à l'Église, attribue à leur leader un pouvoir charismatique surpassant de loin les fonctions qui leur sont attribuées et reconnues par l'Église. Les fidèles désignent bien souvent leur leader par le terme de «prophète » sans se soucier de son statut hiérarchique officiel.

Le problème le plus sérieux se pose lorsque la renommée d'un responsable de camp (pourtant «associé » à l'Eglise : il en affiche la dénomination) s'accroît au point que, dans la perception des fidèles, n'apparaisse plus que la dimension charismatique de leur leader, le «prophète-guérisseur », et que la dénomination de l'Église devienne 
secondaire. A ce stade de développement, il n'est pas rare qu'un tel leader en vienne à former le projet de se séparer de l'Église-mère. Le mode de fonctionnement et de gestion des camps de prière, ainsi que la question du statut de leurs «dirigeants » sont à l'origine de plusieurs dissidences au Ghana comme en Côte-d'Ivoire. Plus globalement, la crise qui aboutit à la dissidence est souvent liée au mode de gestion locale des activités de l'Église (délivrance, camps, etc.) et à la soumission des leaders «charismatiques » à une autorité centralisée. C. Mayrargue ajoute que, dans le contexte béninois, la dépendance des Églises nationales à un siège situé à l'extérieur du pays est l'une des causes principales de dissidence (2001 : 279).

La multiplication des camps en périphérie des assemblées de quartier s'est étendue à la Côte-d'Ivoire, mais non au Burkina Faso. Sur ce point, les dirigeants de l'Église de Pentecôte du Burkina Faso, gênés aussi par le fait que l'inexistence de camps s'accorde avec le faible niveau de développement de l'Église dans ce pays, n'apportent que peu d'éléments de réponse. Certains d'entre eux attribuent la pratique de la délivrance dans les assemblées ivoiriennes et ghanéennes au fait qu'elle y a été volontairement réintroduite pour concurrencer les camps de prière. D'autres évoquent la réticence des fidèles devant des pratiques qu'ils jugent démoniaques et qui les effrayent. Le seul Centre de prière actuellement associé à l'Église de Pentecôte du Burkina Faso est le camp du « vieux Ahmado » de Pellé (Kombissiri) qui correspond davantage à un héritage de la génération antérieure des pasteurs de l'Église, tels que Pinnoaga Yanogo qui en est à l'origine, mais n'a fait l'objet d'aucun investissement de la part de l'Église qui n'entend pas encourager ces pratiques. Ahmado apparaît comme le dernier représentant d'un mouvement que l'Église semble avoir fait avorter. Il reste que la délivrance ne fait pas partie de l'histoire de cette Église au Burkina Faso et que l'Église de Pentecôte n'y subit pas pour l'instant la concurrence d'autres « Ministères de délivrance » si présents dans les pays côtiers. Les autres cas connus de pratique de la délivrance au Burkina Faso sont plutôt associés à l'Église des Assemblées de Dieu et s'organisent autour de quelques leaders, en marge de l'orthodoxie de l'Église. P.-J. Laurent leur consacre une partie de son récent ouvrage (2003), centrée sur quelques portraits de «croyants-guérisseurs » comme Élysée, qui passa une partie de sa vie en Côte-d'Ivoire où il fut converti et qui organise des séances de délivrance collective dans un temple à la périphérie de Ouagadougou. Sur la scène burkinabé, l'évangéliste Mamadou Karambiri, issu des Assemblées de Dieu (de France, où il fut converti durant ses études supérieures) et fondateur du Centre International d'Évangélisation, est aussi très populaire et très influent auprès de la jeune génération de leaders. Comme le personnage d'Élysée, son parcours et ses pratiques le 
placent dans la lignée des nouveaux acteurs religieux hybrides qui avaient émergé au Burkina Faso au milieu des années 1980 et dans toute l'Afrique de l'Ouest, mais ces deux leaders ne sont pas cependant comparables aux leaders de camps qui occupent aujourd'hui la scène pentecôtiste ghanéenne ou ivoirienne. Ni le Centre International d'Évangélisation de Mamadou Karambiri, ni le temple où Elysée exerce ses dons ne sont des structures équivalentes aux camps de prière décrits plus haut. Le décalage entre le Burkina Faso et les pays côtiers reste nettement perceptible.

\section{Conclusion}

L'expansion transnationale et la structure en réseau qui en résulte soumettent l'Église de Pentecôte à des tensions internes qui ébranlent sans cesse ses postures théologiques et politiques les plus fermes. Tout d'abord parce que cette expansion se traduit par la formation d'espaces communautaires marginaux qui échappent bientôt à son contrôle et parce que la centralisation de l'autorité (à Accra) éveille les tentations autonomistes des «agents» locaux qui lui sont soumis. Les plus « charismatiques » de ces agents sont à la fois instrumentalisés par l'Église dans un premier temps pour leur pouvoir attractif, et redoutés, dans un deuxième temps, lorsque leur popularité surpasse celle de la dénomination à laquelle ils appartiennent. Ainsi, les deux espaces marginaux et atypiques que sont les «assemblées en français » et les camps de guérison constituent deux foyers de tensions et de compromis entre l'Église et ses agents «doubles » qui sont aussi deux types d'intermédiaires: les interprètes et les guérisseurs. Ils sont aussi, à la marge, les lieux où l'Église négocie ses ouvertures et ses frontières avec les autres, sur le plan de la langue et dans le traitement des «corps souffrants» où l'Église est confrontée à l'épreuve de l'altérité. Ces structures marginales se présentent comme deux entrées possibles dans l'analyse des politiques d'expansion missionnaire de la Church of Pentecost, dans le sens où les modalités de leur existence sont au centre des modes d'implantation de cette Église à l'étranger. Elles sont également à l'origine des conflits et dissidences qui jalonnent l'histoire de cette Église aussi bien en Afrique qu'en Europe. Ce qui ressort de la circulation au sein de ce réseau d'Église aujourd'hui, c'est moins l'aspect conflictuel de la collaboration missionnaire que la répétition du conflit, dans les mêmes termes, au regard même de l'histoire plus ancienne de cette Église telle qu'elle est décrite par les sources historiques et missionnaires, avec des éléments nouveaux, comme la « crise de la vernacularisation » qui a abouti à la formation des assemblées en langues nationales qui sont elles-mêmes aujourd'hui à l'origine de plusieurs dissidences. 


\section{Bibliographie}

AвAmFo O. A., The Rise of the Charismatic Movement in the mainline Churches in Ghana, Accra, Asempa Publishers, 1993.

Dozon, J.-P., «La Côte d'Ivoire entre démocratie, nationalisme et ethnonationalisme », Politique Africaine ${ }^{\circ} 78,2000$, pp. 45-62.

FANCELLO S., «Au commencement était la dissidence: création et séparation au sein du pentecôtisme ghanéen ", Archives de Sciences Sociales des Religions 122 (avril-juin), 2003, pp. 43-53.

FANCELLO S., «Les politiques identitaires d'une Église Africaine transnationale: The Church of Pentecost (Ghana)», Cahiers d'Études Africaines, à paraître.

GIFFORD P., «Ghana's Charismatic Churches », Journal of Religion in Africa, XXIV (3), 1994, pp. 241-265.

GIFFORD P., African Christianity. Its Public Role, Hurst \& $\mathrm{C}^{\mathrm{ie}}$ London, 1998.

LARBi E.K., Pentecostalism. The Eddies of Ghanaian Christianity, cpcs (Centre of Pentecostal and Charismatic Studies), sapc, series 1, Dansoman, Accra-Ghana, 2001.

LAURENT P.-J., «L'Église des Assemblées de Dieu au Burkina Faso, histoire, transitions et recompositions identitaires », Archives de Sciences sociales des Religions, $\mathrm{n}^{\circ} 105,2001, \mathrm{pp} .71-97$.

LAURENT P.-J., Les pentecôtistes du Burkina Faso. Mariage, pouvoir et guérison, Paris, Karthala, 2003.

LEONARD C., A Giant in Ghana. 3,000 churches in 50 years. The Story of James MkKeown and The Church of Pentecost, New Wine Press, Chichester, 1989.

MAYRARGUE C., «The Expansion of Pentecostalism in Benin : Individual Rationales and Transnational Dynamics » in Corten A., Marshall-Fratani R., Between Babel an d Pentecost. Transnational Pentecostalism in Africa and Latina America, Hurst \& Compagny, London, 2001, pp. 274-292

NTUMY M. K., An Assessment of The Growth and Development of The Church of Pentecost with particular reference to its Impact on the Religious Life of Ghana, Regents Theological College, Nantwich, UK, 2000.

VAN DIJK R., «From Camp to Encompassment: Discourses of Transsubjectivity in the Ghanaian Pentecostal Diaspora », Journal of Religion in Africa, XXVII, 2, 1997, pp. 135-159.

WYLLIE, R. W. «Pioneers of Ghanaian Pentecostalism : Peter Anim and James Mc Kweon », Journal of Religion in Africa, VI, n², 1974, p. 10922. 